\title{
Mining the Prognostic Value of HNRNPAB and Its Function in Breast Carcinoma
}

\author{
Yun Cao $\mathbb{D}$, Wei Zhang $\mathbb{D}$, Yi-Ting Jin $\mathbb{D}$, and Qiang Zou $\mathbb{C}$ \\ Department of General Surgery, Huashan Hospital, Fudan University, Shanghai 200040, China \\ Correspondence should be addressed to Yi-Ting Jin; clara_raky@aliyun.com and Qiang Zou; zouqiang_hs@163.com
}

Received 26 October 2019; Accepted 29 January 2020; Published 14 May 2020

Academic Editor: Atsushi Kurabayashi

Copyright @ 2020 Yun Cao et al. This is an open access article distributed under the Creative Commons Attribution License, which permits unrestricted use, distribution, and reproduction in any medium, provided the original work is properly cited.

\begin{abstract}
Heterogeneous nuclear ribonucleoproteins (HNRNPs) are crucial members in the pathogenesis and progression of numerous cancers. However, the expression pattern and clinical significance of HNRNPs in breast carcinoma (BC) remain to be investigated. In the present study, bioinformatic analysis identified HNRNPAB as the only commonly upregulated HNRNP in BC. Elevated expression of HNRNPAB was positively associated with more aggressive diseases and poorer survival rates in BC. Pathway analysis revealed that HNRNPAB coexpressed genes were enriched in the pathway of G2/M phase transition, and the expression level of HNRNPAB was strongly correlated with those of CCNB1, CDK1, CDC25A, and CDC25C. Experiments in vitro demonstrated that HNRNPAB knockdown suppressed cell proliferation and blocked the G2/M phase transition in BC. Taken together, this study provides the initial evidence that HNRNPAB may be employed as an innovative therapeutic target as well as a prognostic biomarker in BC patients.
\end{abstract}

\section{Introduction}

Breast carcinoma $(\mathrm{BC})$ is the mostly diagnosed tumor and the major cause of cancer-associated mortality among women worldwide [1]. Despite the improved screening, diagnosis and treatment regimens, prognosis for patients with BC remains poor. Therefore, identification of more specific and sensitive biomarkers for early diagnosis and survival prediction, as well as novel therapeutic targets for effective therapy, is of great significance.

Heterogeneous nuclear ribonucleoproteins (HNRNPs) represent a large family of RNA-binding proteins and act as pivotal members in multiple aspects of RNA metabolism [2]. They assist in alternative splicing [3] and polyadenylation of precursor messenger RNA (mRNA) [4, 5], mRNA stability [6], mRNA nuclear export [7], and translational regulation [8-10]. Given their function diversity and complexity, HNRNPs have gained growing interest in disease research. The expressions of HNRNPs are altered in various cancers, suggesting their roles in oncogenesis. HNRNPC modulates the alternative cleavage and polyadenylation profiles in metastatic colon carcinoma [11]. HNRNPQ1 interacts with and enhances the translational efficiency of Aurora-A mRNA, thus contributing to cell proliferation in colorectal carcinoma [12]. HNRNPI regulates neonatal immune adaptation and prevents the development of colorectal carcinoma [13]. Previous studies have reported that HNRNPAB overexpression induces epithelial-mesenchymal transition and promotes the metastasis of hepatocellular carcinoma (HCC) via transcriptional regulation of SNAIL [14] and lncRNAELF209 [15]. HNRNPAB interacts with lncRNA-PCAT19 to activate a subset of cell cycle-related genes in the progression of prostate carcinoma [16]. However, the precise role of HNRNPAB in BC has been blurred.

Herein, a multitude of public datasets and platforms was utilized to determine the commonly upregulated HNRNPs in BC. HNRNPAB was identified as the only upregulated HNRNP in BC samples compared with noncancerous tissues. Higher expression of HNRNPAB indicated poorer survival in BC patients, and its association with clinicopathological characteristics was further analyzed using online databases. Pathway analysis of HNRNPAB coexpressed genes revealed that HNRNPAB might involve in cell cycle regulation, especially the G2/M phase transition. Moreover, 
HNRNPAB expression was strongly correlated with CCNB1, CDK1, CDC25A, and CDC25C expressions. Experiments in vitro confirmed that HNRNPAB knockdown could impede the proliferation capacity of $\mathrm{BC}$ cells and induce the G2/M phase arrest.

\section{Materials and Methods}

2.1. GEPIA Database Analysis. GEPIA (http://gepia.cancerpku.cn/) is an interactive web server for analyzing the RNA sequencing expression data of 9736 tumors and 8587 normal samples from the TCGA and the GTEx projects, using a standard processing pipeline [17]. GEPIA was used to obtain upregulated genes in the TCGA-BRCA database via ANOVA. All overexpressed genes with significance met the criterion of combined $p$ value $<1 E-4$ and log (fold change, FC) $>1$. Spearman's correlations between CCNB1, CDK1, CDC25A, CDC25C, and HNRNPAB were downloaded from the website.

2.2. GEO. The GSE15852 microarray data was obtained from GEO (http://www.ncbi.nlm.nih.gov/geo/). The gene expression profile of 43 pairs of $\mathrm{BC}$ and normal tissue samples was determined. GEO2R was applied to identify all upregulated genes in $\mathrm{BC}$ tissues as opposed to normal breast tissues. All upregulated genes with significance met the criterion of combined $p$ value $<0.05$ and $\log \mathrm{FC}>1$.

2.3. Oncomine Database Analysis. The Oncomine database (https://www.oncomine.org), an online platform that incorporates 715 independent datasets and 86733 samples [18], was utilized to evaluate the expression patterns of HNRNPAB in various tumor samples. The HNRNPAB mRNA level in BC samples was compared with that of their matched normal samples using 8 microarray datasets from 3 cohorts. The fold change of HNRNPAB expression was presented in box plots. The filters and thresholds used to obtain the datasets were set as follows: analysis type: cancer vs. normal analysis; $p$ value: $1 E-04$; FC: 1.5 ; gene rank: $10 \%$, data type: mRNA.

2.4. Breast Cancer Gene-Expression Miner v4.3 (bcGenExMiner v4.3) Analysis. The bc-GenExMiner v4.3 (http://bcgenex.centregauducheau.fr/) is a statistical mining tool of published annotated breast cancer transcriptomic data and RNA-seq [19]. The statistical analyses are grouped in three modules: expression, prognosis, and correlation [20]. The bc-GenExMiner v4.3 correlation module was used to analyze the correlations between HNRNPAB mRNA expression and clinicopathological parameters in $\mathrm{BC}$ patients.

2.5. cBioPortal Database Analysis. The cBioPortal for Cancer Genomics (http://www.cbioportal.org/) is a web resource for exploring, visualizing, and analyzing multiple-dimensional cancer genomics data [21]. The Breast Invasive Carcinoma (TCGA, Provisional) study was selected, and a list of genes correlated with HNRNPAB was acquired from the website. The filters and thresholds used to obtain the gene list were given below: analysis type: mRNA expression (RNA Seq V2
RSEM vs. RNA Seq V2 RSEM); $p$ value: 0.05; Spearman's correlation: 0.5 .

2.6. PrognoScan Database Analysis. The PrognoScan database (http://www.prognoscan.org/) is a web-based platform that evaluates the relationship between candidate gene expression and prognosis in cancer patients [22]. Hazard ratios, 95\% confidence intervals, and Cox $p$ values were automatically calculated by the website.

2.7. Reactome Database Analysis. The Reactome website (http://reactome.ncpsb.org/) provides bioinformatic tools for pathway visualization and interpretation. The core unit of the Reactome data model is the reaction. Entities participating in the reactions form a network of biological interactions and are grouped into pathways [23]. Genes coexpressed with HNRNPAB were assessed using the Reactome Pathway Browser.

2.8. Cell Culture and Transfection. Human BC cell lines MCF7 and MDA-MB-231 were purchased from the Chinese Institute of Biochemistry and Cell Biology (China). MCF7 and MDA-MB-231 cells were maintained in Dulbecco's Modified Eagle's Medium and L-15 Medium (Gibco, USA) with $10 \%$ fetal bovine serum (FBS) (Gibco, USA) at $37^{\circ} \mathrm{C}$ with $5 \% \mathrm{CO}_{2}$, respectively. Lentivirus vectors expressing short hairpin RNA, as well as the scramble controls, were purchased (GeneChem, China) and named as sh-HNRNPAB and sh-NC. The sequence of sh-HNRNPAB was as follows: 5' -CCGGTGAATTGCCAATGGATCCAAACTCGAGTTT GGATCCATTGGCAATTCATTTTTG-3' . Cells were transfected with lentivirus vectors for 48 hours and further selected using $2 \mu \mathrm{g} / \mathrm{mL}$ puromycin (Selleck, USA). The efficiency of HNRNPAB knockdown was confirmed via western blot.

2.9. Western Blot Analysis. Total protein was harvested from cells using RIPA lysis buffer (Beyotime, China). Protein samples were separated by sodium dodecyl-sulfate polyacrylamide (SDS-PAGE) gel and transferred to polyvinylidene fluoride (PVDF) membranes. Blots were washed and incubated with antibodies. The protein bands were then visualized with an ECL detection system. The primary and secondary antibodies utilized in this study were listed as follows: anti-GAPDH (60004-1-Ig, Proteintech, USA), anti-HNRNPAB Abcam, USA), anti-CDK1 (ab133327, Abcam, USA), anti-Cyclin B1 (ab32053, Abcam, USA), anti-CDC25A (sc-7389, Santa Cruz, USA), anti-CDC25C (ab32444, Abcam, USA), anti-ER (ab75635, Abcam, USA), anti-HER2 (ab134182, Abcam, USA), HRP-conjugated goat anti-mouse IgG (SA00001-1, Proteintech, USA), and HRP-conjugated goat anti-rabbit IgG (SA00001-2, Proteintech, USA).

2.10. Cell Proliferation Assay. Cell growth was detected with cell count kit-8 (CCK8) following the manufacturer's instruction (Yeasen, China). For the CCK8 assay, $1 \times 10^{3}$ cells were seeded per well in quintuplicate in 96 well plates. After 1, 2, 3, 4, and 5 days, cells were changed with $100 \mu \mathrm{L}$ fresh medium added $10 \mu \mathrm{L}$ CCK8 reagent and incubated for 



(a)

(b)
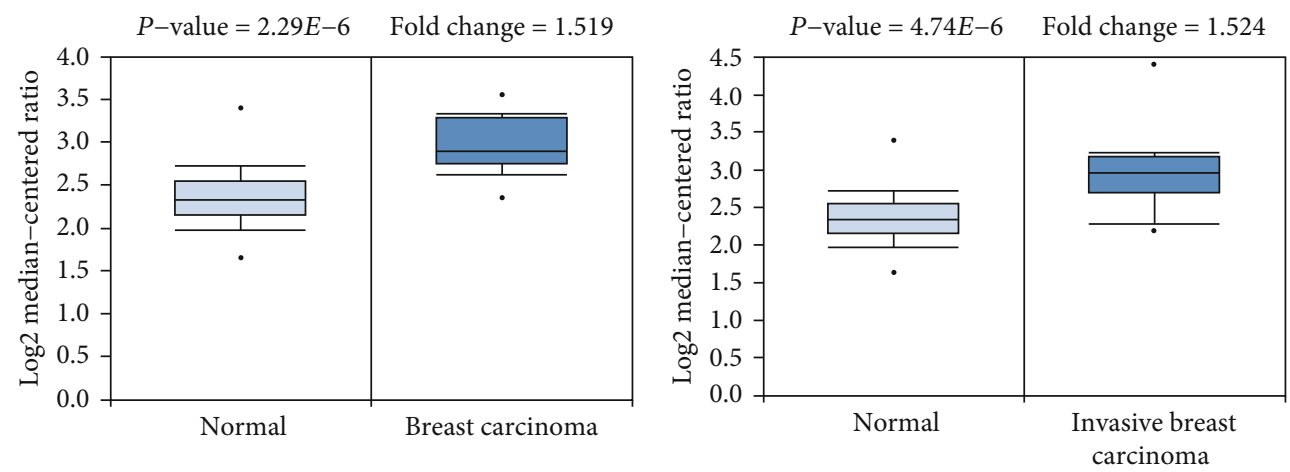

(c)

(d)

Figure 1: Continued. 


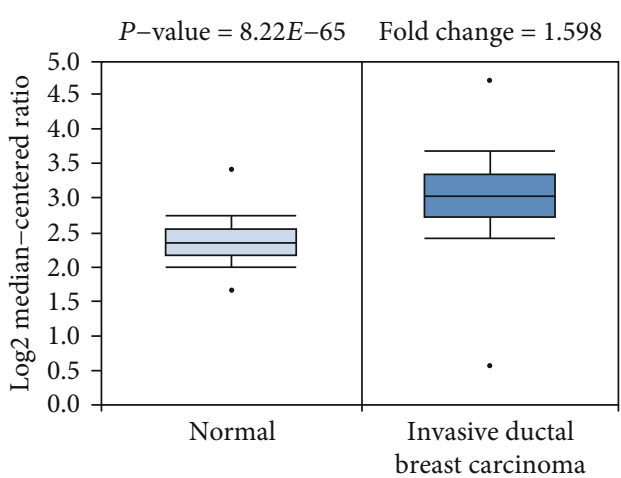

(e)

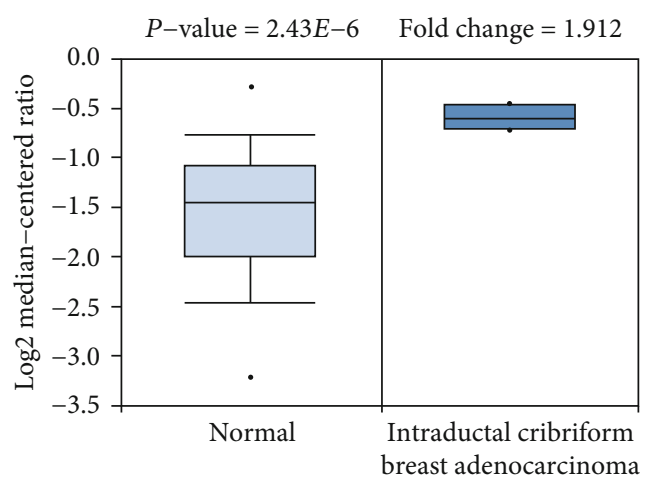

(g)

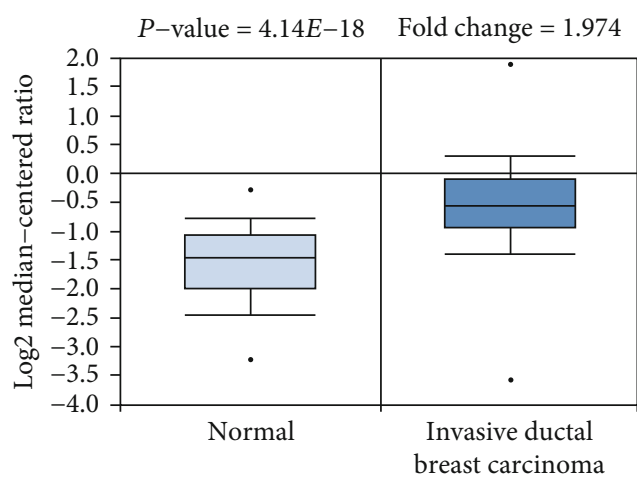

(i)

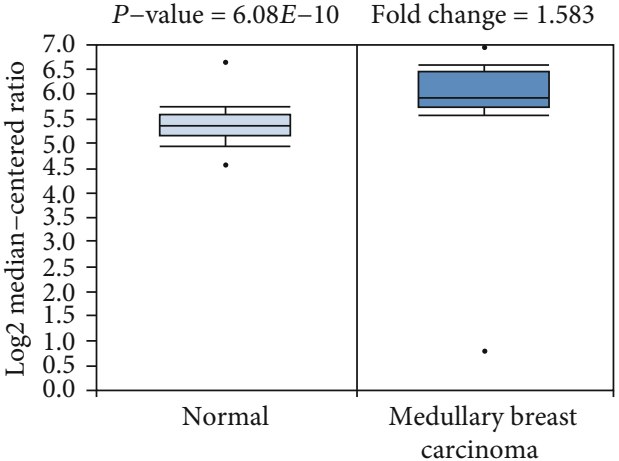

(f)

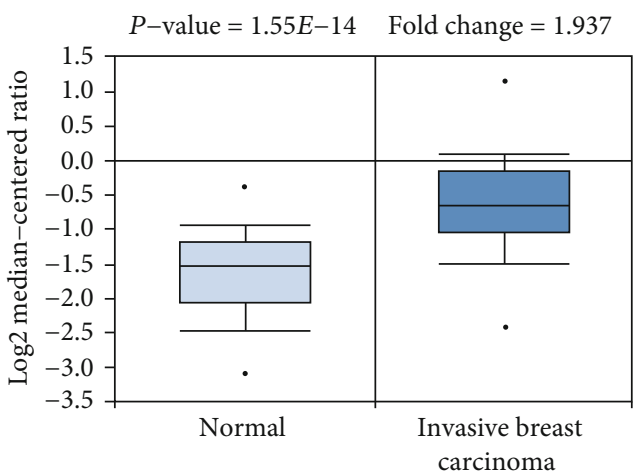

(h)

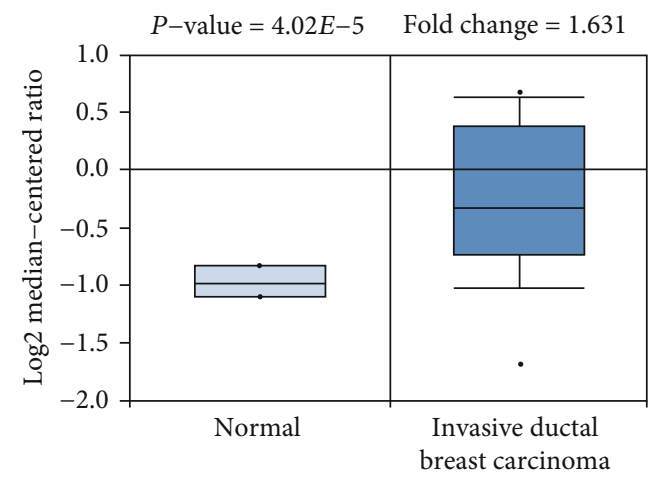

(j)

\section{HNRNPAB $\quad 6 \%$ " \\ Genetic alteration \\ Amplification \\ Deep deletion \\ mRNA High mRNA Low No alterations - Not profiled}

(k)

FIGURE 1: Upregulation of HNRNPAB in BC tissues. (a) Venn diagram of commonly upregulated HNRNPs in TCGA-BRCA and GSE15852. HNRNPAB was identified as the only overexpressed HNRNP in BC tissues compared to noncancerous tissues. (b) HNRNPAB was significantly upregulated in various malignancies including BC. (c-j) Box plots comparing the HNRNPAB mRNA level in normal tissues (left) and BC tissues (right) were generated from the Oncomine database. (k) HNRNPAB was amplified or upregulated in 6\% of BC cases.

2 hours at $37^{\circ} \mathrm{C}$. Absorbance of each well was measured at $450 \mathrm{~nm}$ at indicated time points.

2.11. Flow Cytometry. Transfected cells were starved for 24 hours and then maintained in fresh medium with 10\% FBS for 24 hours. Cells were harvested and fixed in ice-cold $75 \%$ ethanol overnight at $4^{\circ} \mathrm{C}$. Then, cells were suspended in PI/R-
Nase staining buffer (BD Biosciences, USA) for 15 minutes in the dark. Cell cycle distribution was assessed using a flow cytometer (BD Biosciences, USA).

2.12. Statistical Analysis. Each experiment was performed for three times. Two-tailed Student's $t$-test was employed for in vitro data. Data were presented as means \pm standard 
TABLE 1: Oncomine analysis of HNRNPAB expression in BC.

\begin{tabular}{|c|c|c|c|c|c|}
\hline No. & Cohort name & Data type & Sample (n) & Fold change & $p$ value \\
\hline \multirow{4}{*}{1} & \multirow{4}{*}{ Curtis Breast Statistics } & mRNA & Breast carcinoma (14) vs. normal (144) & 1.519 & $2.29 E-06$ \\
\hline & & mRNA & Invasive breast carcinoma (21) vs. normal (144) & 1.524 & $4.74 E-06$ \\
\hline & & mRNA & Invasive ductal breast carcinoma (1556) vs. normal (144) & 1.598 & $8.22 E-65$ \\
\hline & & mRNA & Medullary breast carcinoma (32) vs. normal (144) & 1.583 & $6.08 E-10$ \\
\hline \multirow{3}{*}{2} & \multirow{3}{*}{ TCGA Breast Statistics } & mRNA & Intraductal cribriform breast adenocarcinoma (3) vs. normal (61) & 1.912 & $2.43 E-06$ \\
\hline & & mRNA & Invasive breast carcinoma (76) vs. normal (61) & 1.937 & $1.55 E-14$ \\
\hline & & mRNA & Invasive ductal breast carcinoma (389) vs. normal (61) & 1.974 & $4.14 E-18$ \\
\hline 3 & Zhao Breast Statistics & mRNA & Invasive ductal breast carcinoma (35) vs. normal (3) & 1.631 & $4.02 E-05$ \\
\hline
\end{tabular}

deviation (SD) and analyzed with GraphPad Prism 7.0 software (La Jolla, USA). $p$ value $<0.5$ was considered astatistically significant.

\section{Results}

3.1. Identification of Upregulated HNRNPs in BC Tissues. To identify overexpressed HNRNPs in BC, all upregulated genes in $\mathrm{BC}$ tissues versus noncancerous tissues were obtained from TCGA-BRCA database and GSE15852. 1406 overexpressed genes were found $(\log F C>1.5, p<0.0001)$ in TCGA-BRCA, while 344 upregulated genes were determined $(\log F C>1.5, p<0.05)$ in GSE15852. The Venn diagram revealed that HNRNPAB was the only HNRNP among the commonly upregulated genes in TCGA-BRCA and GSE15852 (see Figure 1(a)). Oncomine database analysis further confirmed that HNRNPAB was overexpressed in several solid tumors and hematological malignancies (see Figure 1(b)). In particular, HNRNPAB mRNA was statistically higher in $\mathrm{BC}$ tissues compared with that in matched normal tissues (see Figures 1(c)-1(j)). Details of HNRNPAB expression in these datasets are listed in Table 1 . The cBioPortal database analysis demonstrated that HNRNPAB was amplified or upregulated in $6 \%$ of BC cases (see Figure 1(k)). Accordingly, the above lines of results suggested that HNRNPAB might act as an oncogene in the development of BC.

3.2. Clinical Significance of HNRNPAB in Patients with BC. To unveil the associations between HNRNPAB expression and patients' clinicopathological characteristics, data from bc-GenExMiner v4.3 was obtained and analyzed using Welch's $t$-test (see Table 2). With regards to age, HNRNPAB mRNA expression was higher in younger patients $(p=0.0029$, see Figure 2(a)). Furthermore, BC patients with a positive nodal status exhibited higher HNRNPAB mRNA expression compared with those with negative nodal status $(p<0.0001$, see Figure 2(b)). Estrogen (ER) status and progesterone $(\mathrm{PR})$ status were negatively correlated with the HNRNPAB mRNA level $(p<0.0001$, see Figures $2(c)$ and $2(d))$. Conversely, HNRNPAB mRNA expression was prominently increased in patients with positive HER2 status $(p<0.0001$, see Figure 2(e)). Patients with triple-negative BC (TNBC) exhibited higher HNRNPAB mRNA expression than non-TNBC patients $(p<0.0001$, see Figure 2(f)). Our
TABLE 2: The associations between HNRNPAB mRNA expression and clinicopathological parameters in BC.

\begin{tabular}{|c|c|c|c|}
\hline Variable & Case & mRNA expression & $p$ value \\
\hline Age (years) & & & 0.0029 \\
\hline$\leq 51$ & 1099 & $\uparrow$ & \\
\hline$>51$ & 3209 & - & \\
\hline Nodal status & & & $<0.0001$ \\
\hline Positive & 1646 & $\uparrow$ & \\
\hline Negative & 2416 & - & \\
\hline ER (IHC) & & & $<0.0001$ \\
\hline Positive & 3911 & - & \\
\hline Negative & 552 & $\uparrow$ & \\
\hline PR (IHC) & & & $<0.0001$ \\
\hline Positive & 3498 & - & \\
\hline Negative & 829 & $\uparrow$ & \\
\hline HER2 (IHC) & & & $<0.0001$ \\
\hline Positive & 661 & $\uparrow$ & \\
\hline Negative & 3582 & - & \\
\hline Triple-negative status & & & $<0.0001$ \\
\hline Not TNBC & 4119 & - & \\
\hline TNBC & 317 & $\uparrow$ & \\
\hline Basal-like status & & & $<0.0001$ \\
\hline Not basal-like & 3837 & - & \\
\hline Basal-like & 832 & $\uparrow$ & \\
\hline SBR grade & & & $<0.0001$ \\
\hline SBR1 & 544 & - & \\
\hline SBR2 & 1699 & $\uparrow$ & \\
\hline SBR3 & 1374 & $\uparrow$ & \\
\hline NPI grade & & & $<0.0001$ \\
\hline NPI1 & 1173 & - & \\
\hline NPI2 & 1525 & $\uparrow$ & \\
\hline NPI3 & 416 & $\uparrow$ & \\
\hline
\end{tabular}

Note: ER: estrogen receptor; PR: progesterone receptor; HER2: human epidermal growth factor receptor 2; IHC: immunochemistry; TNBC: triplenegative breast carcinoma; SBR: Scarf-Bloom-Richardson; NPI: Nottingham prognostic index.

results also found that the HNRNPAB mRNA level was upregulated in patients with basal-like BC, comparing with those without basal-like BC $(p<0.0001$, see Figure $2(\mathrm{~g}))$. In 


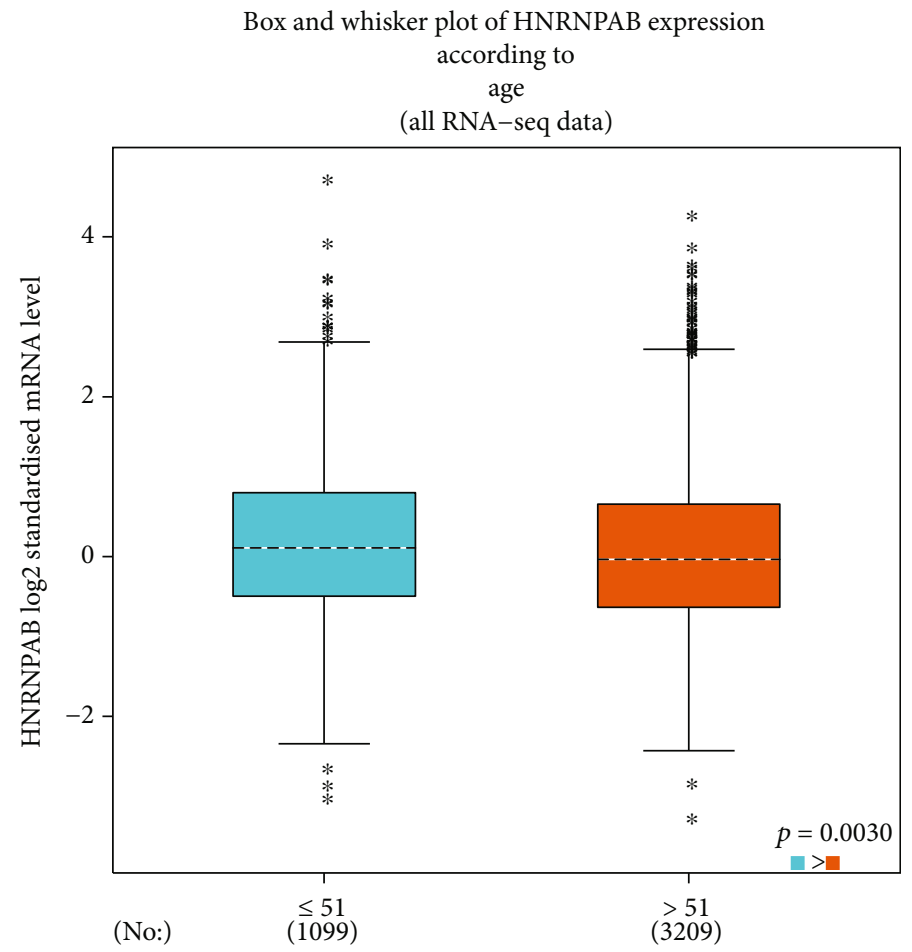

(a)

Box and whisker plot of HNRNPAB expression according to nodal status (all RNA-seq data)

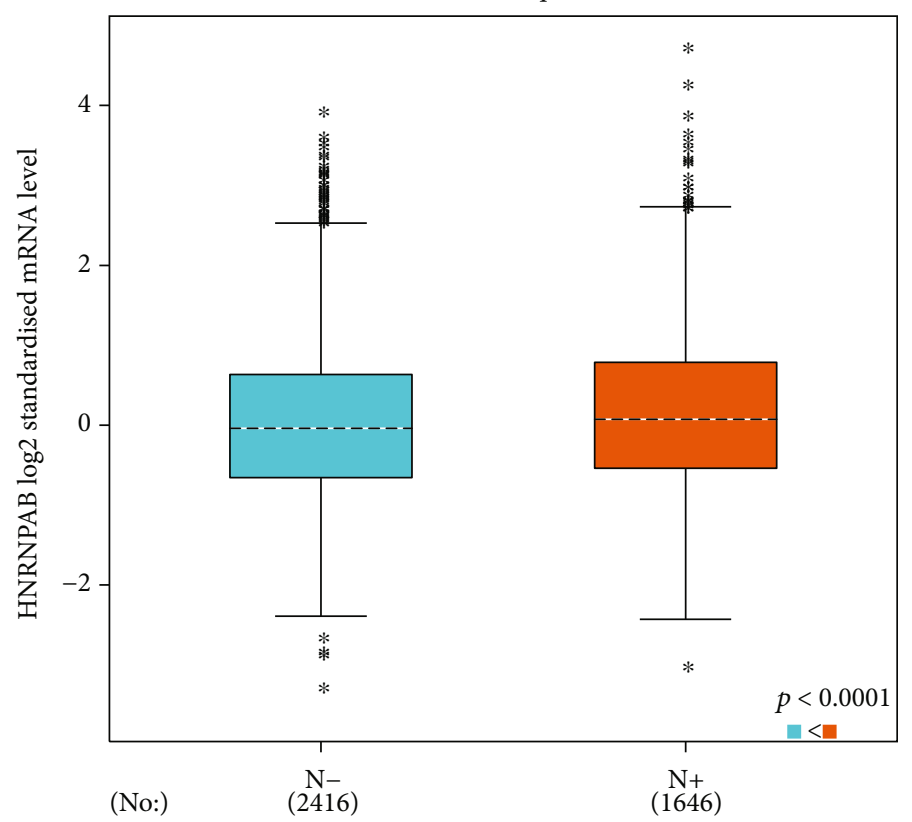

(b)

FIgURe 2: Continued. 


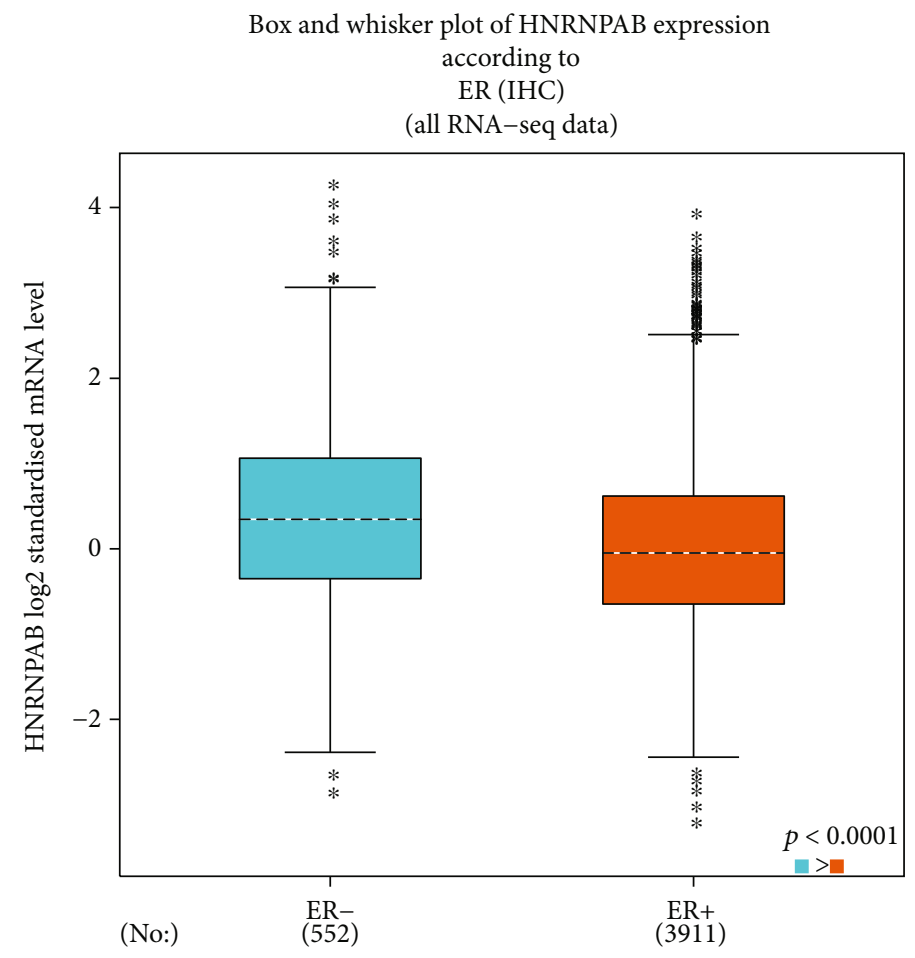

(c)

Box and whisker plot of HNRNPAB expression according to PR (IHC)

(all RNA-seq data)

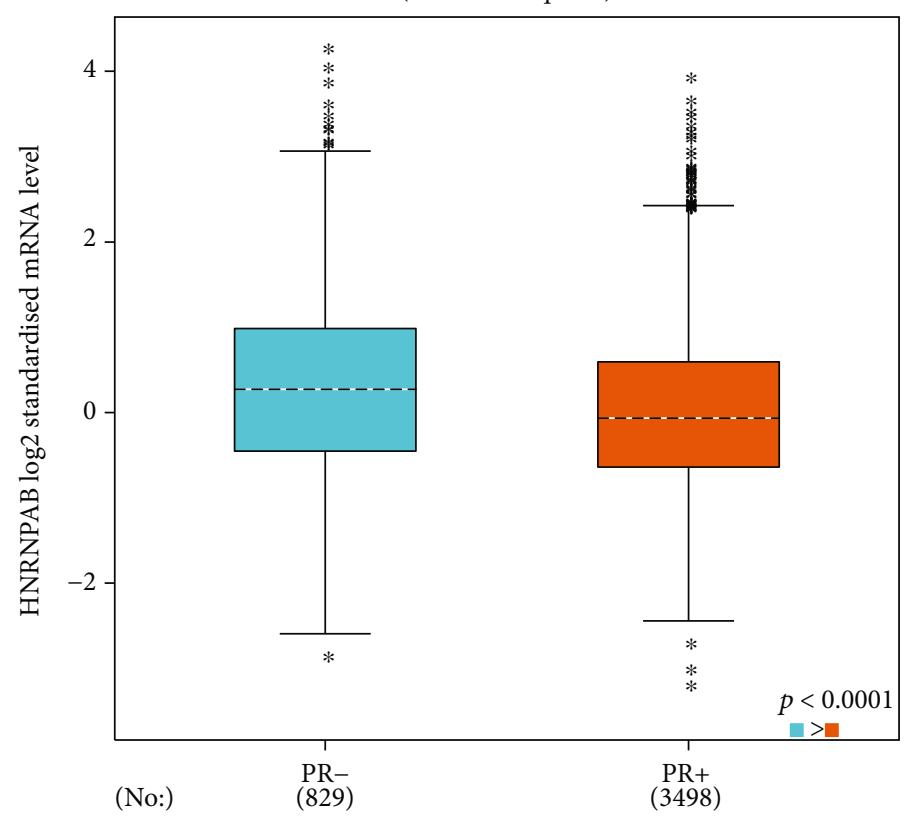

(d)

Figure 2: Continued. 


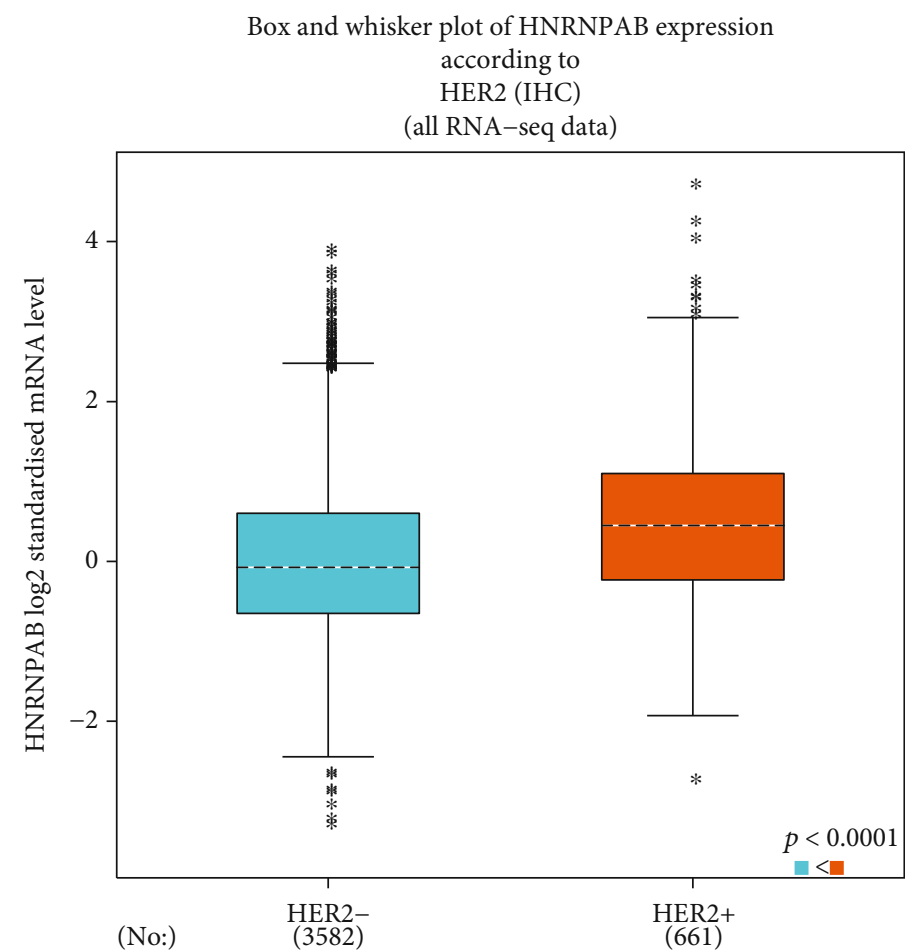

(e)

Box and whisker plot of HNRNPAB expression according to triple-negative status (all RNA-seq data)

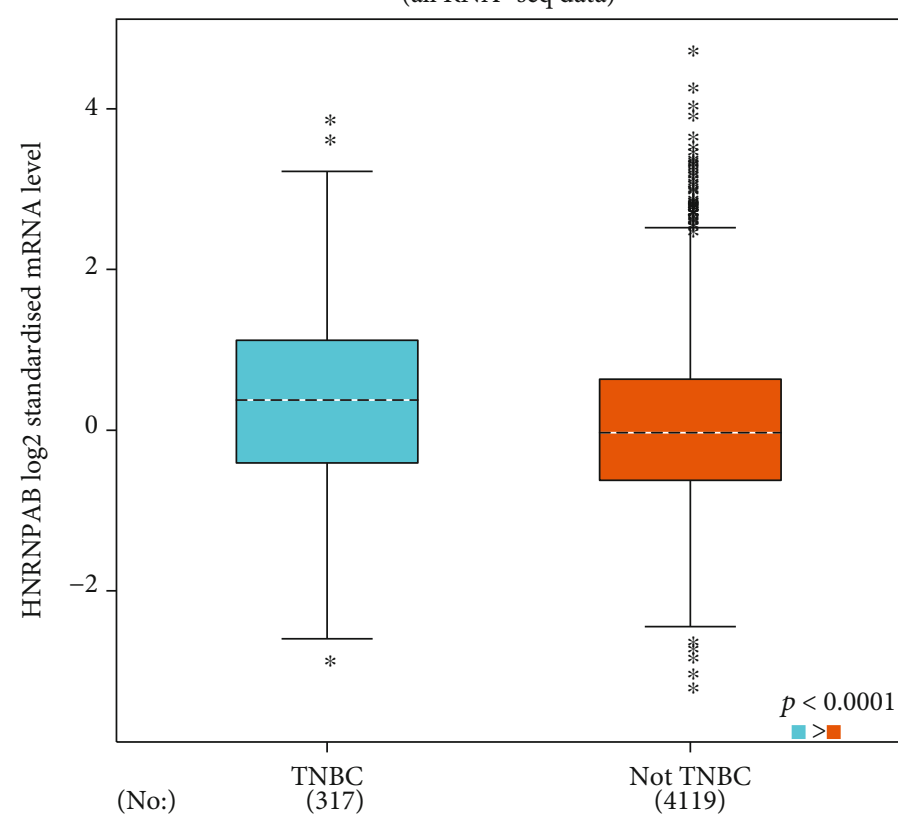

(f)

Figure 2: Continued. 


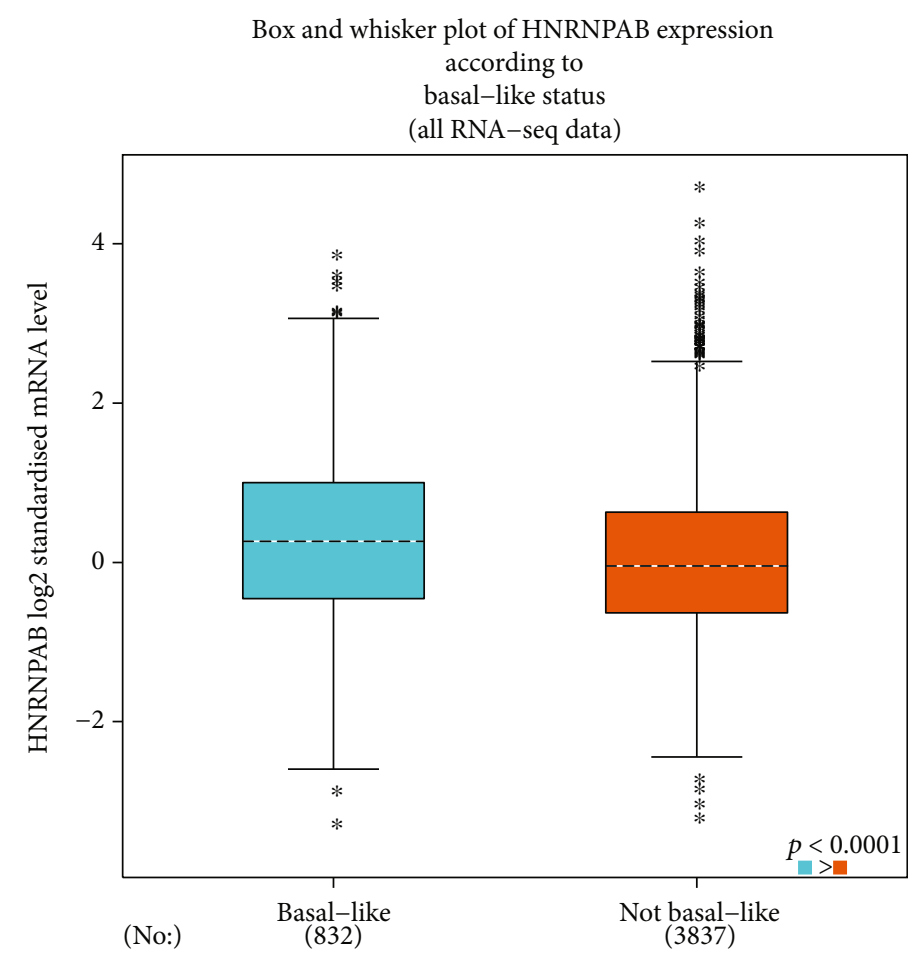

(g)

Box and whisker plot of HNRNPAB expression according to

SBR

(all RNA-seq data)

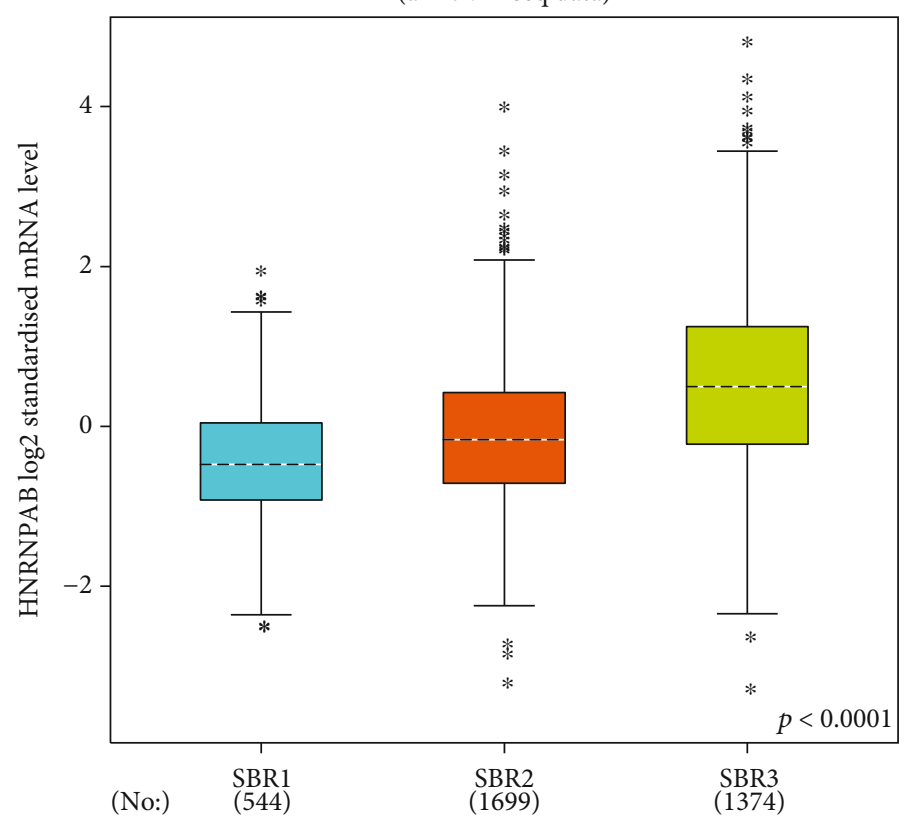

(h)

Figure 2: Continued. 


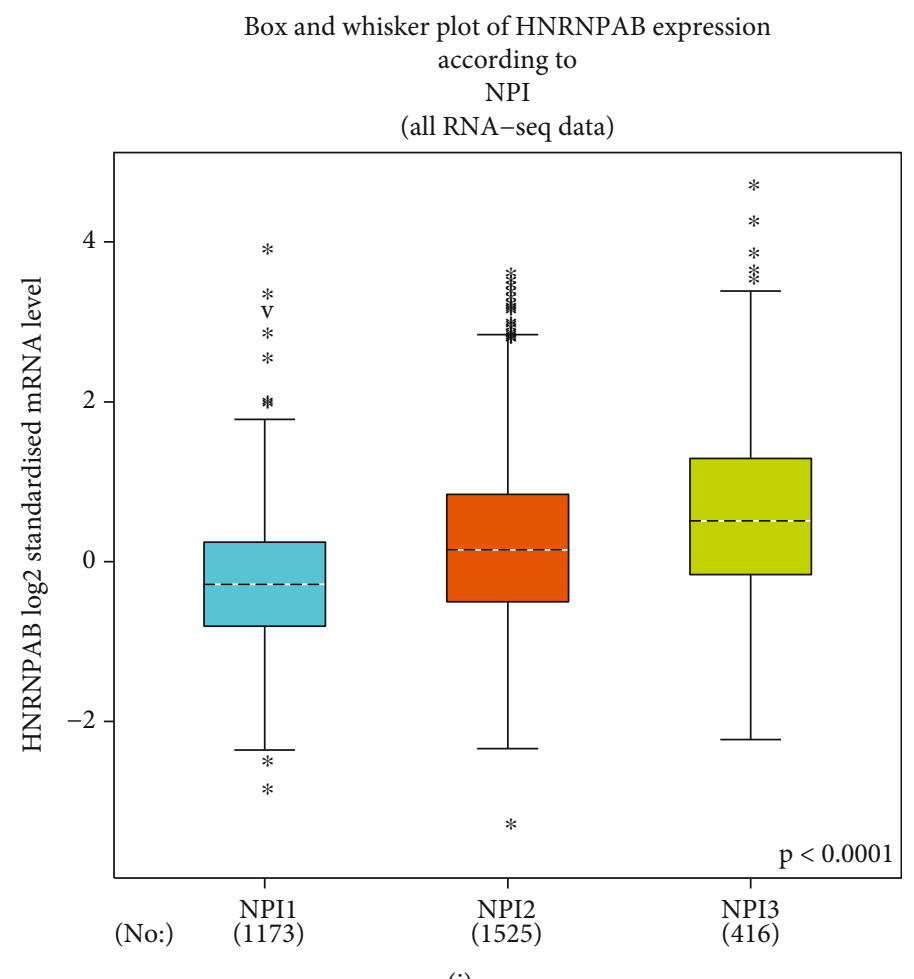

(i)

FIGURE 2: The correlations between HNRNPAB expression and clinicopathological parameters in BC patients were evaluated using bcGenExMiner v4.3. Global significant difference between groups was determined using Welch's $t$-test to calculate $p$ values, following with the Dunnett-Tukey-Kramer $t$-test.

terms of Scarff-Bloom-Richardson (SBR) grade and Nottingham prognostic index (NPI) grade, higher expression of HNRNPAB was significantly associated with more advanced SBR grade and NPI grade $(p<0.0001$, see Figures $2(\mathrm{~h})$ and 2(i)).

3.3. High HNRNPAB Expression Is Associated with Poor Prognosis in Patients with BC. The prognostic value of HNRNPAB in patients with $B C$ was evaluated using the PrognoScan website. Survival curve analysis illustrated that the higher HNRNPAB level was remarkably correlated with shorter relapse-free survival (RFS), overall survival (OS), distant metastasis-free survival (DMFS), disease-free survival (DFS), and disease-specific survival (DSS) in BC, thus suggesting that HNRNPAB expression might be predictive of clinical outcomes in BC patients (see Figure 3 and Table 3).

3.4. The Potential Pathways of HNRNPAB in the Development of $B C$. To clarify the biological functions and signaling pathways of HNRNPAB in tumorigenesis, pathway enrichment analysis of HNRNPAB-associated genes from TCGA-BRCA was performed using the Reactome database. The analysis identified the top 25 pathways in which HNRNPAB might be involved, including cell cycle and G2/M phase transition (see Figure 4(a) and Table 4). Furthermore, HNRNPAB mRNA expression was strongly correlated with CCNB1 $(R=0.76 ; p=1.3 \mathrm{E}-257), \mathrm{CDC} 25 \mathrm{C}(R=0.74$; $p=2.6 \mathrm{E}-242)$, CDK1 $(R=0.7 ; p=7 \mathrm{E}-206)$, and CDC25A
$(R=0.67 ; \quad p=9.5 \mathrm{E}-183) \quad$ mRNA expressions (see Figure 4(b)). Cyclin B1, cyclin-dependent kinase 1 (CDK1), cell division cycle 25A (CDC25A), and cell division cycle 25C (CDC25C) were G2/M-phase-related proteins, indicating that HNRNPAB might participate in the regulation of $\mathrm{G} 2 / \mathrm{M}$ phase transition in $\mathrm{BC}$.

3.5. HNRNPAB Exerts Promotive Effects on BC Cell Proliferation and Cell Cycle Progression. To determine the biological functions of HNRNPAB in BC progression, HNRNPAB was stably knockdown in MCF7 and MDAMB-231 cells. The decreased expression of HNRNPAB was verified via western blot (see Figure 5(a)). The CCK8 assay demonstrated that HNRNPAB knockdown significantly suppressed the growth of BC cells (see Figure 5(b)). Flow cytometry was further conducted to investigate the effect of HNRNPAB on BC cell cycle distribution. Cell cycle analysis revealed that HNRNPAB knockdown led to an accumulation of cells in the G2/M phase, while the percentage of cells entering the G1 phase was reduced (see Figure 5(c)). Cyclin $\mathrm{B} 1, \mathrm{CDK} 1, \mathrm{CDC} 25 \mathrm{~A}$, and CDC25C are key regulators indispensable for $\mathrm{G} 2 / \mathrm{M}$ phase transition. CDK1/Cyclin B1 complex triggers mitosis via phosphorylation of multiple substrates, while $\mathrm{CDC} 25 \mathrm{~A} / \mathrm{C}$ are protein phosphatases responsible for activation of CDK1. Western blot analysis confirmed that the protein levels of Cyclin B1, CDK1, CDC25A, and CDC25C were decreased in HNRNPABknockdown cells (see Figure 5(d)). These results were consistent with those of flow cytometry, indicating that HNRNPAB 

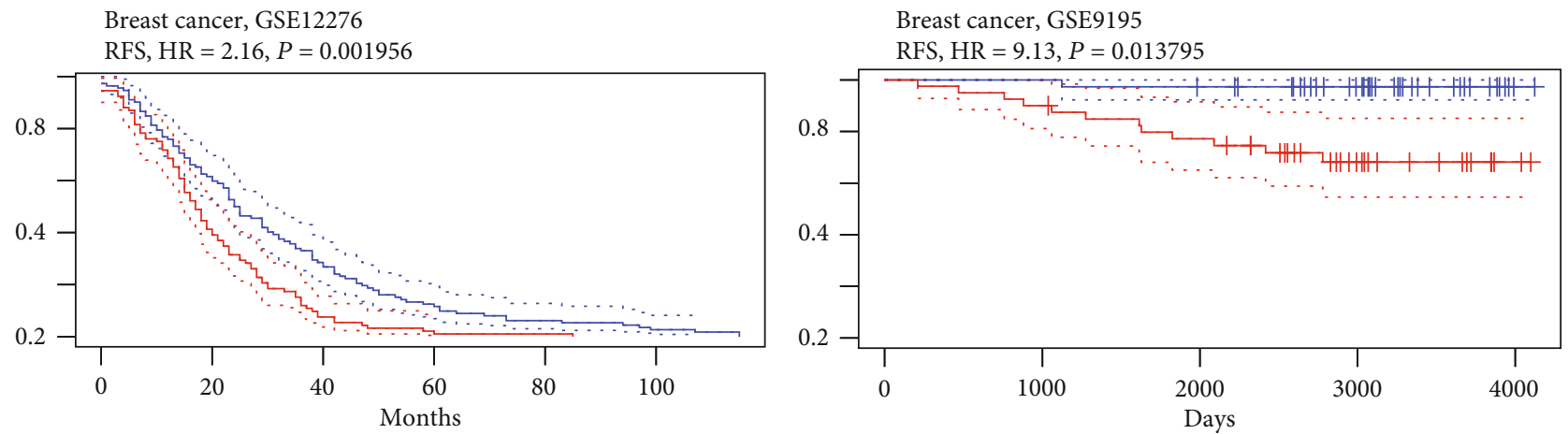

High $n=92$
Low $n=112$

High $n=40$
Low $n=37$

(a)
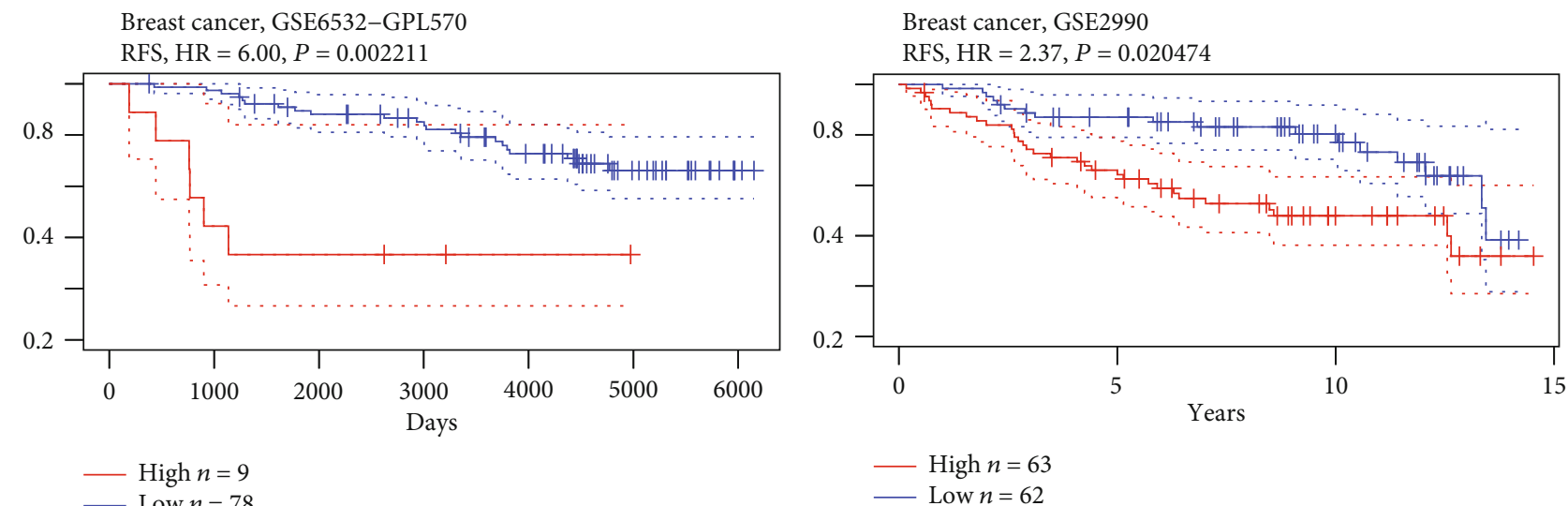

(c)

(d)

Breast cancer, GSE1456-GPL96

RFS, HR $=4.01, P=0.013795$

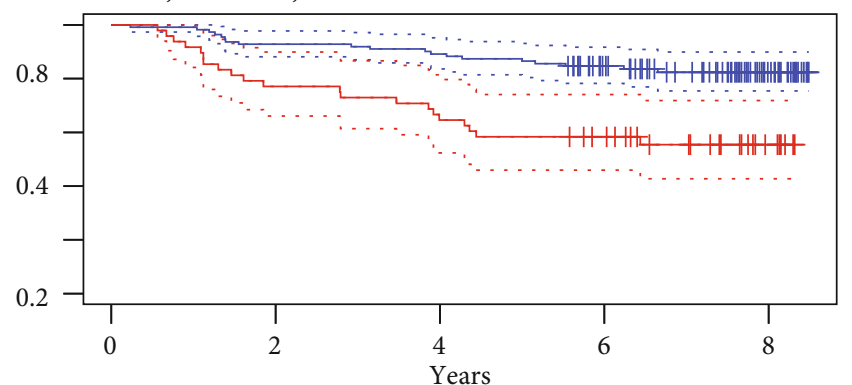

OS, $\mathrm{HR}=5.84, P=0.000183$

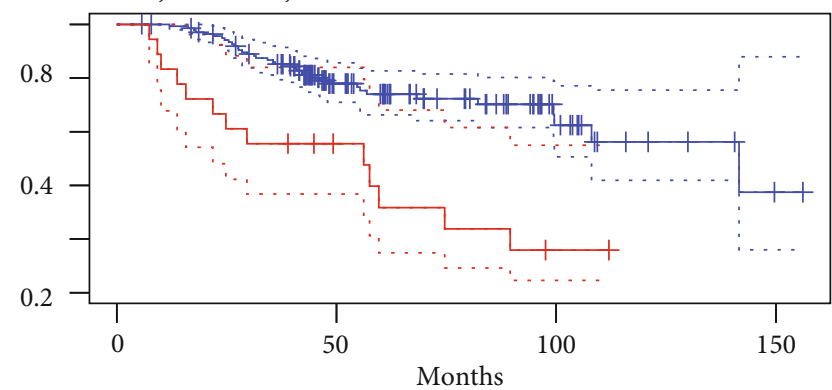

- High $n=48$

- High $n=18$

Low $n=111$

(e)

FIgURe 3: Continued. 


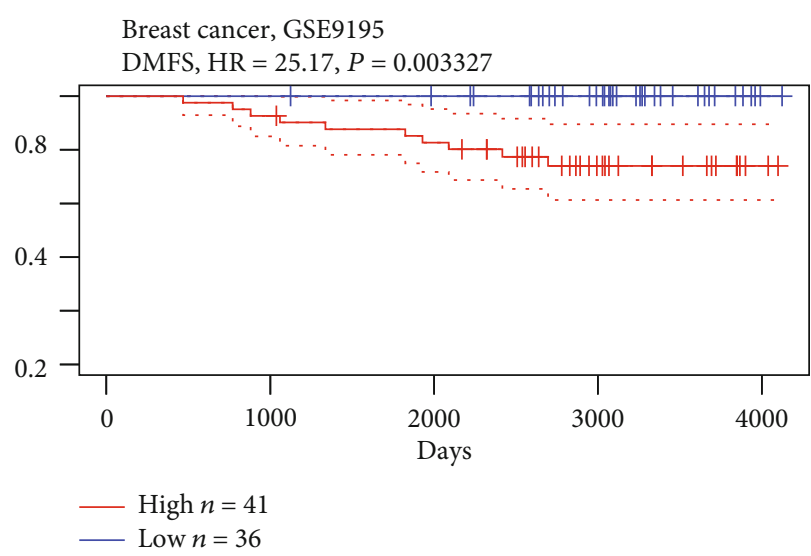

(g)

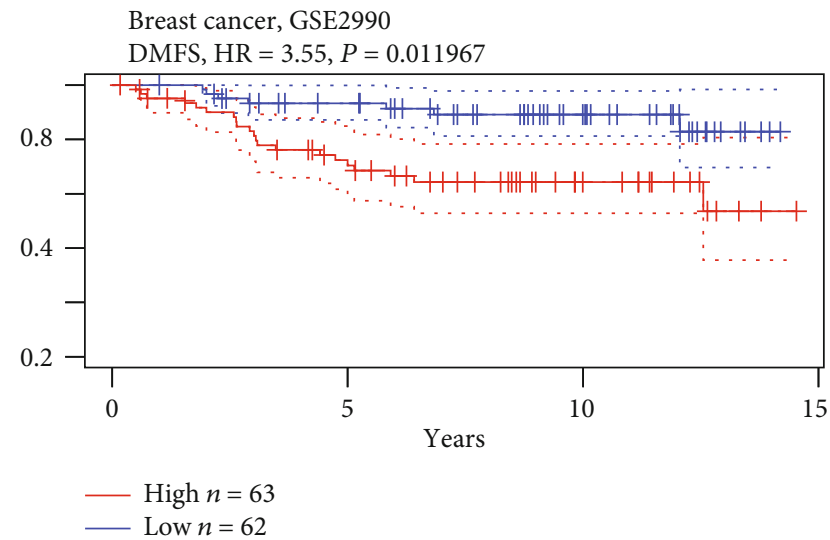

(i)

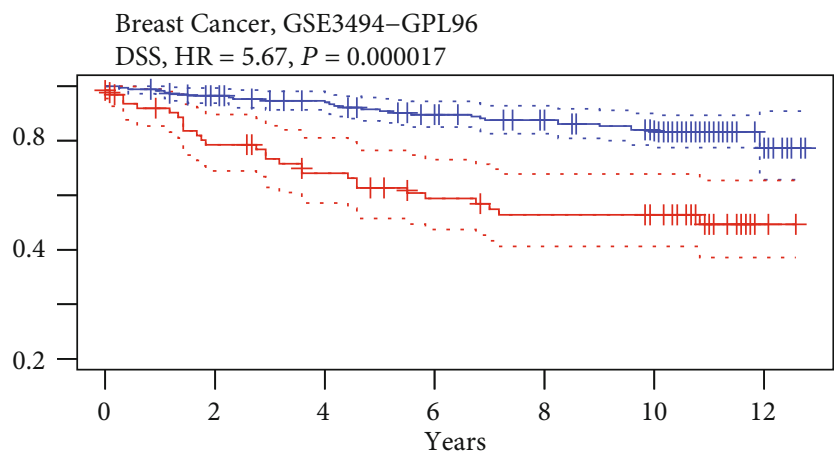

High $n=64$
Low $n=172$

(k)

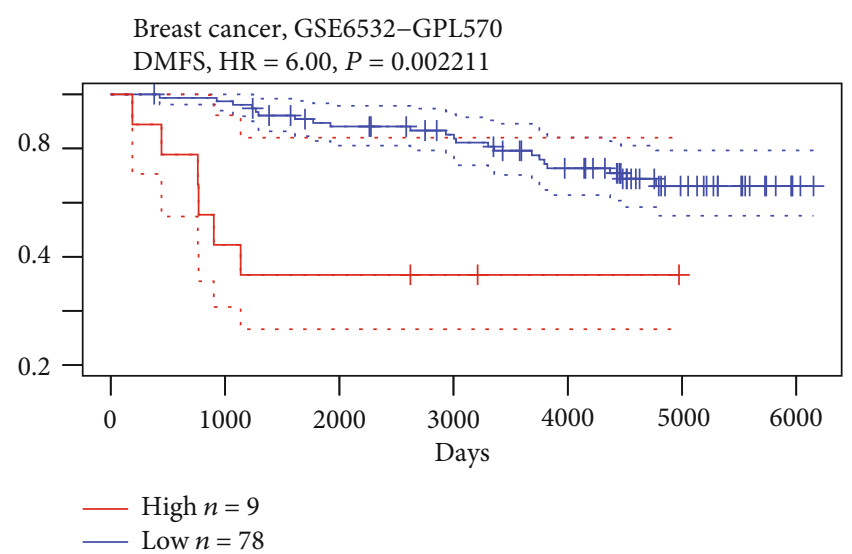

(h)

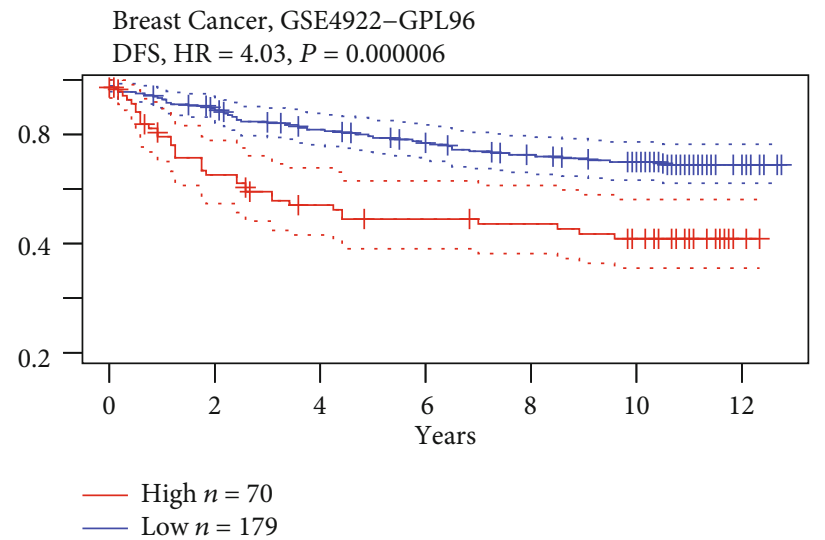

(j)

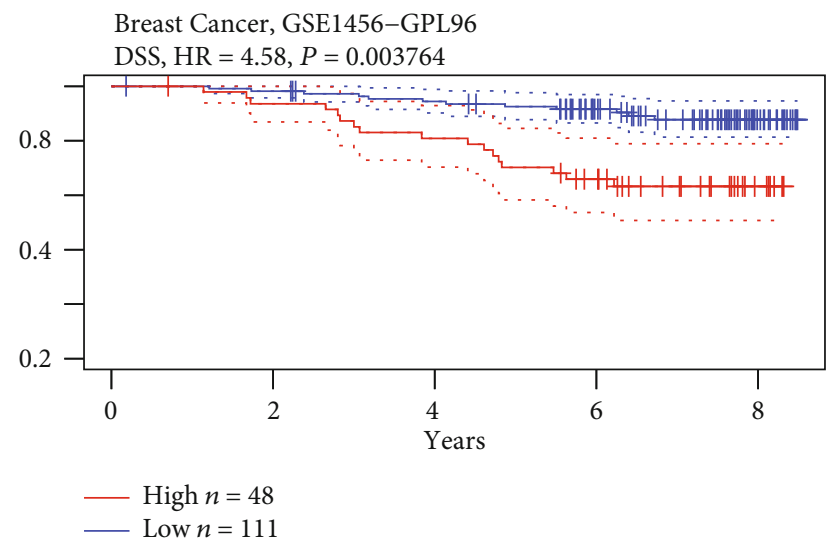

(l)

FIGURE 3: The prognostic role of HNRNPAB in BC patients. Survival curve analyses were performed with PrognoScan datasets. Hazard ratios, 95\% confidence intervals, and Cox $p$ values were automatically obtained from the website. Higher expression of HNRNPAB indicated unfavorable outcomes in $\mathrm{BC}$ patients.

knockdown brought about the G2/M phase arrest in breast cancer cells.

\section{Discussion}

$\mathrm{BC}$ is the most common malignant tumor among women worldwide [1]. Despite the great achievements in screening, diagnosis and therapy for BC, novel therapeutic targets as well as predictive indicators are urgently required. The
HNRNP family members are frequently altered in numerous malignancies and act as crucial players in cancer occurrence and progression [24]. However, the expression pattern and prognostic value of HNRNPs in BC have yet to be elucidated.

The present study screened out the commonly upregulated HNRNPs in TCGA-BRCA and GSE15852 using the Venn diagram. HNRNPAB was identified as the only overexpressed HNRNP in BC tissues relative to normal breast tissues. Zhou et al. reported that worse OS and a higher 
TABLE 3: The expression of HNRNPAB and survival rates in BC patients.

\begin{tabular}{lcccccc}
\hline Dataset & Endpoint & Probe ID & Sample number & Cox $p$ value & HR & 95\% CI \\
\hline GSE4922-GPL96 & Disease free survival & 201277_s_at & 249 & 0.000006 & 4.03 & $2.21-7.36$ \\
GSE3494-GPL96 & Disease specific survival & 201277_s_at & 236 & 0.000017 & 5.67 & $2.57-12.51$ \\
GSE3143 & Overall survival & 38094_at & 158 & 0.000183 & 5.84 & $2.32-14.72$ \\
GSE1456-GPL96 & Relapse free survival & 201277_s_at & 159 & 0.001363 & 4.01 & $1.71-9.39$ \\
GSE12276 & Relapse free survival & 201277_s_at & 204 & 0.001956 & 2.16 & $1.33-3.51$ \\
GSE6532-GPL570 & Relapse free survival & 201277_s_at & 87 & 0.002211 & 6.00 & $1.90-18.89$ \\
GSE6532-GPL570 & Distant metastasis free survival & 201277_s_at & 87 & 0.002211 & 6.00 & $1.90-18.89$ \\
GSE9195 & Distant metastasis free survival & 201277_s_at & 77 & 0.003327 & 25.17 & $2.92-216.77$ \\
GSE1456-GPL96 & Disease specific survival & 201277_s_at & 159 & 0.003764 & 4.58 & $1.64-12.81$ \\
GSE2990 & Distant metastasis free survival & 201277_s_at & 125 & 0.011967 & 3.55 & $1.32-9.53$ \\
GSE9195 & Relapse free survival & 201277_s_at & 77 & 0.013795 & 9.13 & $1.57-53.10$ \\
GSE2990 & Relapse free survival & 201277_s_at & 125 & 0.020474 & 2.37 & $1.14-4.93$ \\
\hline
\end{tabular}

Note: HR: hazard ratio; CI: confidence interval.

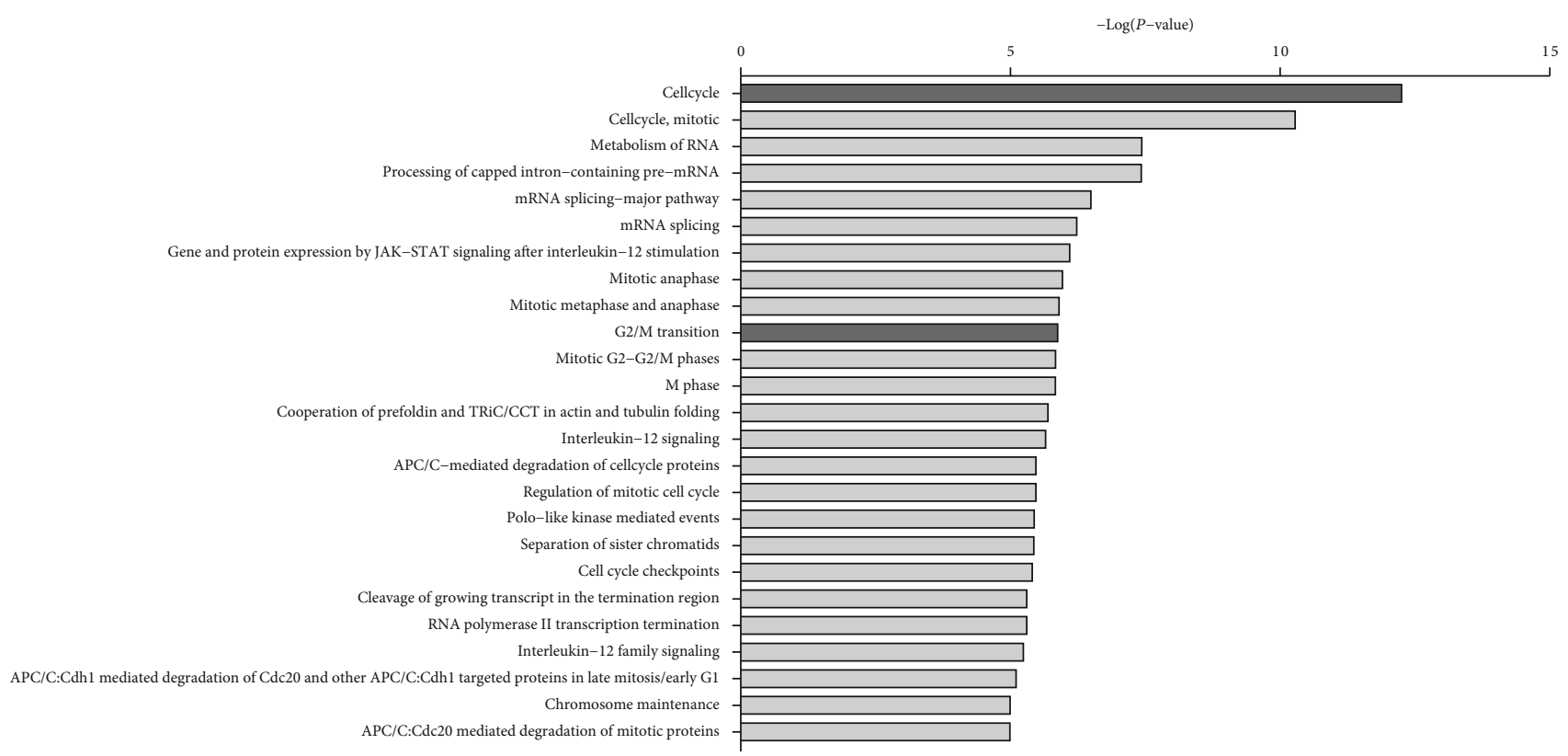

(a)
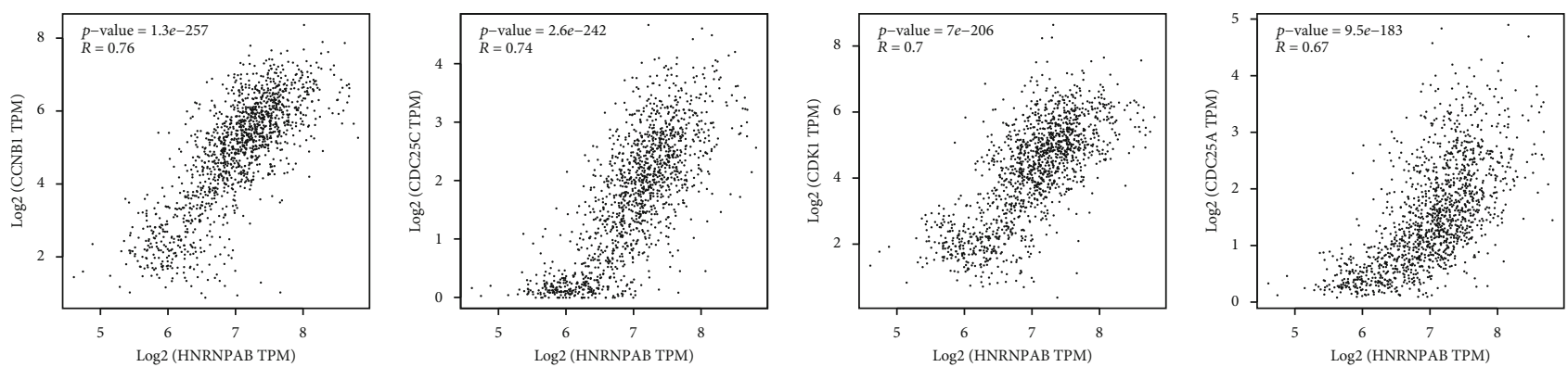

(b)

FIGURE 4: Pathway enrichment analysis of HNRNPAB coexpressed genes using Reactome platform. (a) HNRNPAB coexpressed genes were highly enriched in the pathways of cell cycle and G2/M phase transition. (b) The mRNA level of HNRNPAB was strongly correlated with those of CCNB1, CDK1, CDC25A, and CDC25C. 
TABLE 4: Pathway analysis of HNRNPAB-associated genes in Reactome database.

\begin{tabular}{lccccc}
\hline No. & Top 25 pathways in Reactome database & No. of input genes & $p$ value & $-\log (p$ value $)$ & FDR \\
\hline 1 & Cell cycle & $31 / 682$ & $5.59 E-13$ & 12.25258819 & $3.03 E-10$ \\
2 & Cell cycle, mitotic & $26 / 570$ & $5.22 E-11$ & 10.2823295 & $1.41 E-08$ \\
3 & Metabolism of RNA & $26 / 782$ & $3.66 E-08$ & 7.436518915 & $5.05 E-06$ \\
4 & Processing of capped intron-containing pre-mRNA & $15 / 256$ & $3.74 E-08$ & 7.427128398 & $5.05 E-06$ \\
5 & mRNA splicing-major pathway & $12 / 185$ & $3.20 E-07$ & 6.494850022 & $3.46 E-05$ \\
6 & mRNA splicing & $12 / 196$ & $5.85 E-07$ & 6.232844134 & $5.27 E-05$ \\
7 & Gene and protein expression by & $8 / 74$ & $7.94 E-07$ & 6.100179498 & $6.11 E-05$ \\
8 & Mitotic anaphase & $12 / 208$ & $1.08 E-06$ & 5.966576245 & $6.61 E-05$ \\
9 & JAK-STAT signaling after interleukin-12 stimulation & $12 / 211$ & $1.25 E-06$ & 5.903089987 & $6.61 E-05$ \\
10 & Mitotic metaphase and anaphase & $12 / 212$ & $1.32 E-06$ & 5.879426069 & $6.61 E-05$ \\
11 & G2/M transition & $12 / 214$ & $1.45 E-06$ & 5.838631998 & $6.61 E-05$ \\
12 & Mitotic G2-G2/M phases & $16 / 390$ & $1.47 E-06$ & 5.832682665 & $6.61 E-05$ \\
13 & M phase & $6 / 37$ & $2.01 E-06$ & 5.696803943 & $8.24 E-05$ \\
14 & Cooperation of prefolding and & $8 / 85$ & $2.20 E-06$ & 5.657577319 & $8.36 E-05$ \\
15 & TRiC/CCT in actin and tubulin folding & $8 / 90$ & $3.34 E-06$ & 5.476253533 & $1.09 E-04$ \\
16 & Interleukin-12 signaling & $8 / 90$ & $3.34 E-06$ & 5.476253533 & $1.09 E-04$ \\
17 & APC/C-mediated degradation of cell cycle proteins & $5 / 23$ & $3.62 E-06$ & 5.441291429 & $1.09 E-04$ \\
18 & Regulation of mitotic cell cycle & $11 / 194$ & $3.64 E-06$ & 5.438898616 & $1.09 E-04$ \\
19 & Polo-like kinase-mediated events & $13 / 279$ & $3.91 E-06$ & 5.407823243 & $1.09 E-04$ \\
20 & Separation of sister chromatids & $7 / 67$ & $4.93 E-06$ & 5.307153081 & $1.23 E-04$ \\
21 & Cell cycle checkpoints & $7 / 67$ & $4.93 E-06$ & 5.307153081 & $1.23 E-04$ \\
22 & Cleavage of growing transcript in the termination region & $8 / 97$ & $5.74 E-06$ & 5.241088108 & $1.38 E-04$ \\
23 & RNA polymerase II transcription termination & $7 / 72$ & $7.84 E-06$ & 5.105683937 & $1.80 E-04$ \\
24 & Interleukin-12 family signaling & $8 / 105$ & $1.01 E-05$ & 4.995678626 & $2.14 E-04$ \\
25 & APC/C:Cdh1-mediated degradation of Cdc20 and & $7 / 75$ & $1.02 E-05$ & 4.991399828 & $2.14 E-04$ \\
\hline
\end{tabular}

recurrence rate were observed in HCC patients with high HNRNPAB expression [14], suggesting that HNRNPAB served as a predictive indicator for HCC. Using the PrognoScan website, it was demonstrated that high HNRNPAB expression was significantly related with unfavorable RFS, OS, DMFS, DFS, and DSS in BC. Patient's age, ER status, and $\mathrm{PR}$ status were found to be negatively correlated to the HNRNPAB mRNA level. Conversely, nodal metastasis, HER2 status, TNBC and basal-like status, and SBR and NPI grades were positively associated with HNRNPAB expression. Collectively, these findings supported the role of HNRNPAB as a powerful predictor of poor patient outcomes in multiple malignancies including BC.

Given the aforementioned evidences that HNRNPAB is a potential oncogenic agent in $\mathrm{BC}$, we therefore investigated the precise mechanisms of HNRNPAB in regulating malignant biological properties of $\mathrm{BC}$ cells. Pathway enrichment analysis of HNRNPAB coexpressed genes demonstrated that cell cycle was the top enriched pathway, following with RNA metabolism and the JAK-STAT signaling pathway, which were closely related with the occurrence and progression of $\mathrm{BC}$.
The role of HNRNPAB in mediating BC cell aggressiveness was further evaluated in vitro. The CCK8 assay clearly revealed that downregulation of HNRNPAB could significantly repress the proliferation of $\mathrm{BC}$ cells. Hua et al. demonstrated that HNRNPAB interacted with the long isoform of lncRNA-PCAT19 and promoted cell growth in prostate carcinoma [16], which agrees with our data. We further performed flow cytometry to analyze the effect of HNRNPAB on cell cycle and revealed that HNRNPAB knockdown blocked the G2/M phase transition in BC cells. Western blot analysis confirmed that Cyclin B1, CDK1, CDC25A, and CDC25C were decreased in HNRNPAB-knockdown cells. These results were consistent with those of pathway analysis. Taken together, our observations implied that upregulation of HNRNPAB caused enhanced aggressiveness of BC cells; however, further studies on the underlying mechanisms are required.

\section{Conclusions}

This study was the first to illustrate that HNRNPAB was the only HNRNP commonly upregulated in BC tissues 


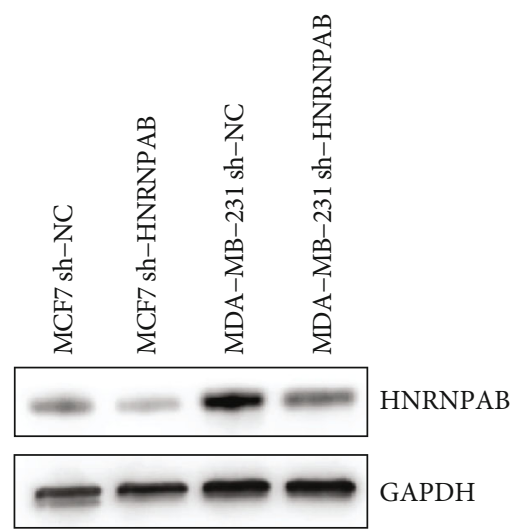

(a)
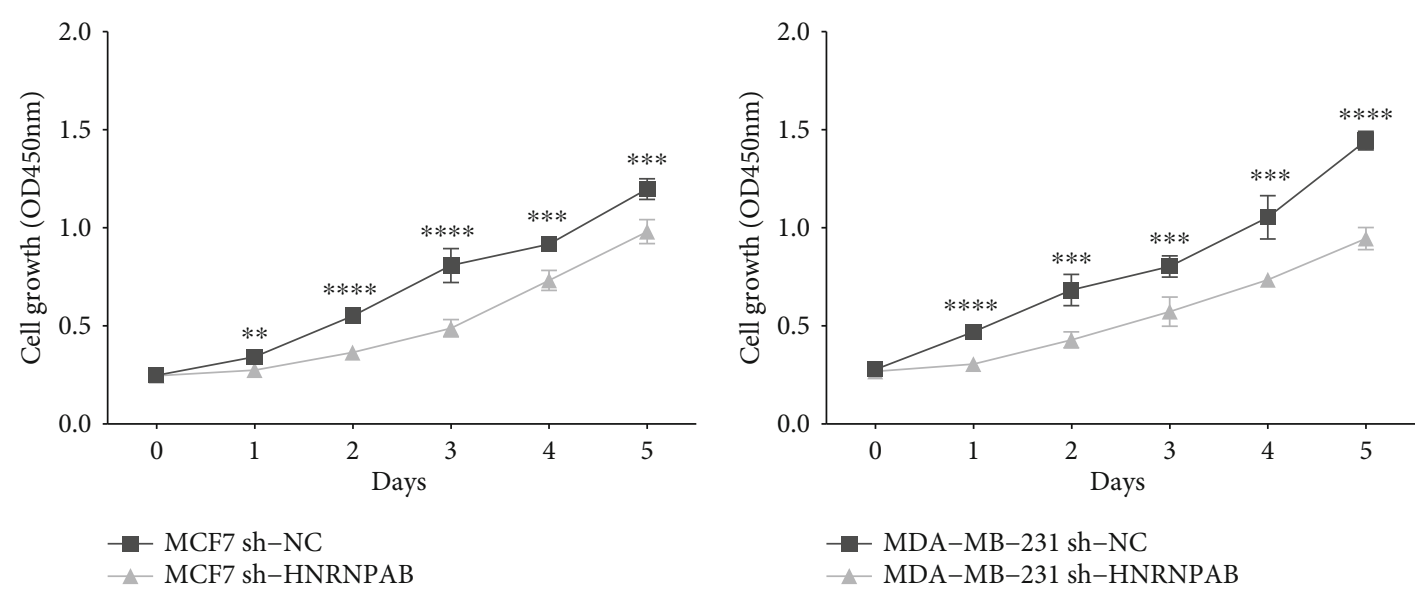

(b)

Figure 5: Continued. 
MCF7 sh-NC

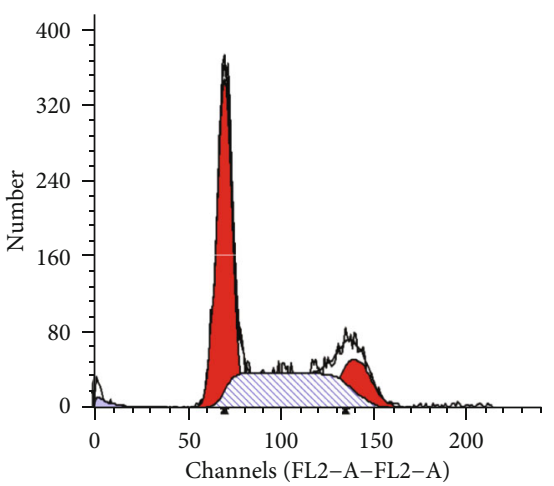

MDA-MB-231 sh-NC

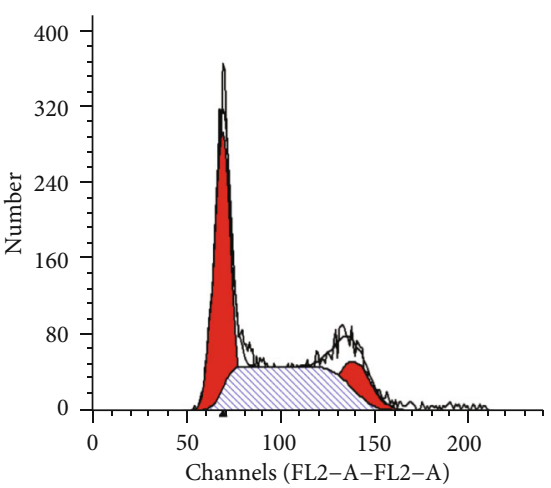

MCF7 sh-HNRNPAB

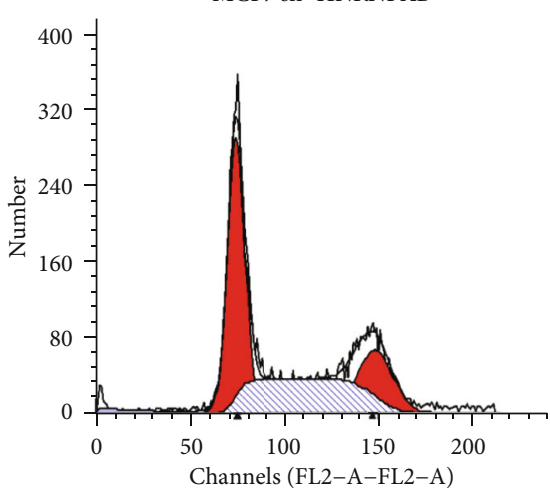

MDA-MB-231 sh-HNRNPAB
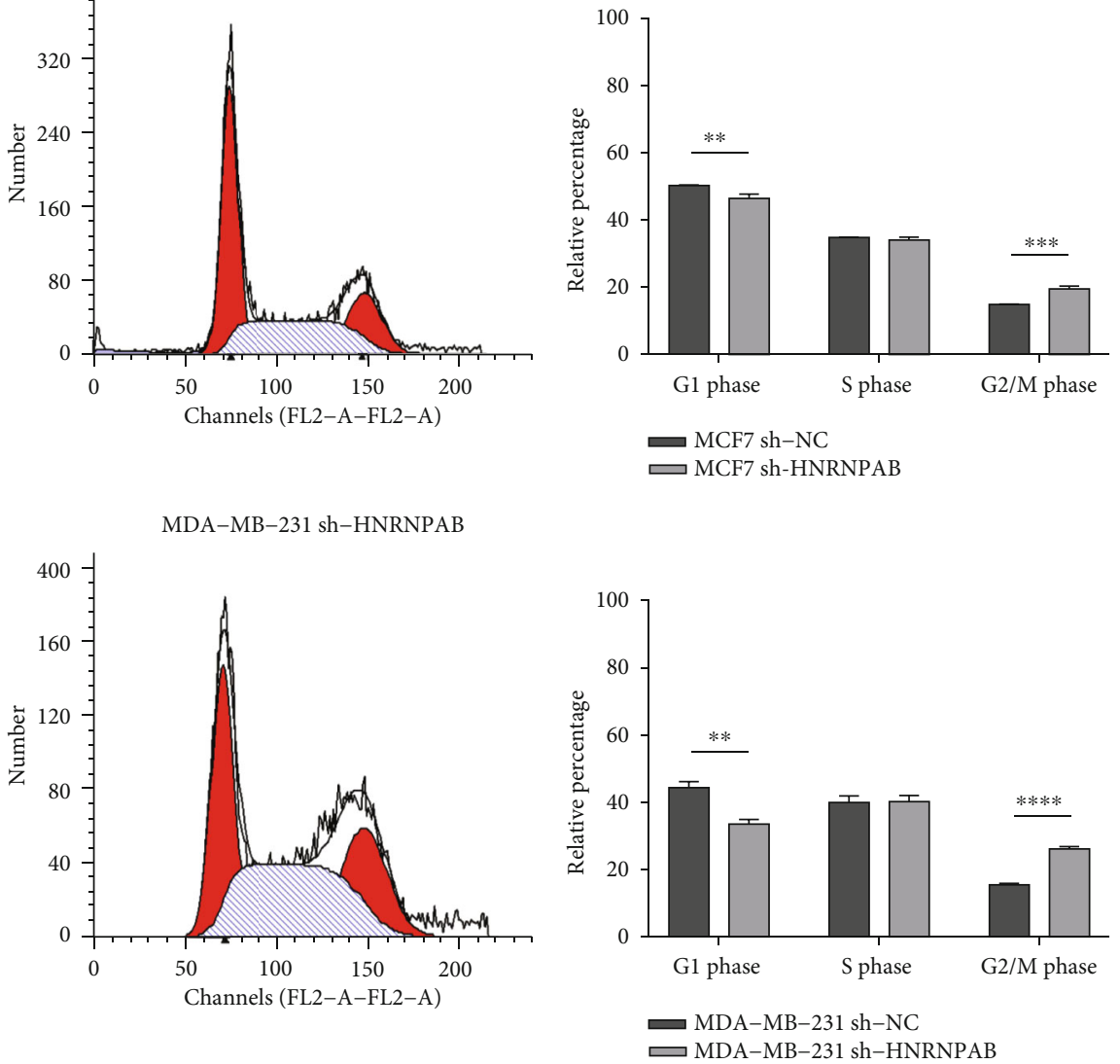

(c)

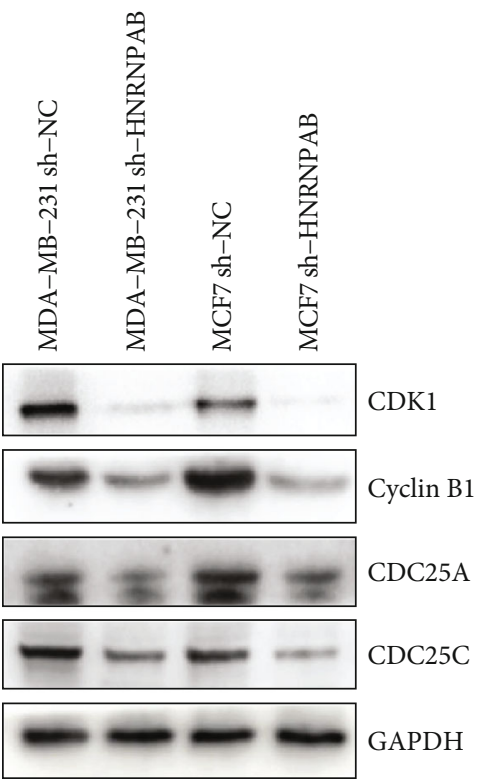

(d)

FIGURE 5: Experiments in vitro revealed the promotive effect of HNRNPAB on BC cells. (a) Western blot analysis of the efficiency of HNRNPAB knockdown. (b) HNRNPAB knockdown impeded the growth of BC cells. (c) HNRNPAB knockdown caused G2/M phase arrest in BC cells. (d) Western blot analysis of key regulatory molecules in G2/M phase transition in indicated BC cells. Data were shown as means $\pm \mathrm{SD},{ }^{* *} p<0.01 ;{ }^{* * *} p<0.001 ;{ }^{* * * *} p<0.0001$. 
compared with adjacent normal tissues. High HNRNPAB expression was associated with unfavorable clinical outcomes in patients with BC. The HNRNPAB expression level was negatively correlated with patient's age, ER status, and $\mathrm{PR}$ status and positively associated with nodal, HER2, TNBC, and basal-like status. Additionally, HNRNPAB expression was increased in patients with more advanced SBR and NPI grades. Furthermore, bioinformatic analysis revealed that HNRNPAB might be involved in G2/M phase transition and was strongly associated with CCNB1, CDK1, CDC25A, and $\mathrm{CDC} 25 \mathrm{C}$. Experiments in vitro demonstrated that HNRNPAB knockdown suppressed the cell proliferation capacity and impeded the G2/M phase transition. To summarize, this study sheds new light on better understanding the fundamental role of HNRNPs in BC progression and discovers HNRNPAB as an innovative therapeutic target and prognostic biomarker for $\mathrm{BC}$ patients.

\section{Data Availability}

The datasets used and/or analyzed in the current study are available from the corresponding author upon reasonable request.

\section{Conflicts of Interest}

The authors claimed that they had no conflicts of interest.

\section{Authors' Contributions}

Yun Cao and Wei Zhang contributed equally to this work.

\section{References}

[1] F. Bray, J. Ferlay, I. Soerjomataram, R. L. Siegel, L. A. Torre, and A. Jemal, "Global cancer statistics 2018: GLOBOCAN estimates of incidence and mortality worldwide for 36 cancers in 185 countries," CA: a Cancer Journal for Clinicians, vol. 68, no. 6, pp. 394-424, 2018.

[2] G. Dreyfuss, M. J. Matunis, S. Pinol-Roma, and C. G. Burd, "hnRNP proteins and the biogenesis of mRNA," Annual Review of Biochemistry, vol. 62, no. 1, pp. 289-321, 1993.

[3] Y. D. Choi, P. J. Grabowski, P. A. Sharp, and G. Dreyfuss, "Heterogeneous nuclear ribonucleoproteins: role in RNA splicing," Science, vol. 231, no. 4745, pp. 1534-1539, 1986.

[4] X. Ji, J. Wan, M. Vishnu, Y. Xing, and S. A. Liebhaber, “ $\alpha \mathrm{CP}$ Poly $(\mathrm{C})$ binding proteins act as global regulators of alternative polyadenylation," Molecular and Cellular Biology, vol. 33, no. 13, pp. 2560-2573, 2013.

[5] Y. Yoon, M. C. McKenna, D. A. Rollins et al., "Anxiety-associated alternative polyadenylation of the serotonin transporter mRNA confers translational regulation by hnRNPK," Proceedings of the National Academy of Sciences of the United States of America, vol. 110, no. 28, pp. 11624-11629, 2013.

[6] C. Ren, S. J. Cho, Y. S. Jung, and X. Chen, "DNA polymerase $\eta$ is regulated by poly(rC)-binding protein 1 via mRNA stability," The Biochemical Journal, vol. 464, no. 3, pp. 377-386, 2014.

[7] A. McCloskey, I. Taniguchi, K. Shinmyozu, and M. Ohno, "hnRNP C tetramer measures RNA length to classify RNA polymerase II transcripts for export," Science, vol. 335, no. 6076, pp. 1643-1646, 2012.
[8] E. K. Lee, H. H. Kim, Y. Kuwano et al., "hnRNP C promotes APP translation by competing with FMRP for APP mRNA recruitment to P bodies," Nature Structural \& Molecular Biology, vol. 17, no. 6, pp. 732-739, 2010.

[9] Y. V. Svitkin, A. Yanagiya, A. E. Karetnikov et al., "Control of translation and miRNA-dependent repression by a novel poly(A) binding protein, hnRNP-Q," PLoS Biology, vol. 11, no. 5, article e1001564, 2013.

[10] M. Majumder, I. Yaman, F. Gaccioli et al., "The hnRNAbinding proteins hnRNP L and PTB are required for efficient translation of the Cat-1 arginine/lysine transporter mRNA during amino acid starvation," Molecular and Cellular Biology, vol. 29, no. 10, pp. 2899-2912, 2009.

[11] H. Fischl, J. Neve, Z. Wang et al., "hnRNPC regulates cancerspecific alternative cleavage and polyadenylation profiles," Nucleic Acids Research, vol. 47, no. 14, pp. 7580-7591, 2019.

[12] C. H. Lai, Y. C. Huang, J. C. Lee et al., "Translational upregulation of_Aurora-A_by hnRNP Q1 contributes to cell proliferation and tumorigenesis in colorectal cancer," Cell Death \& Disease, vol. 8, no. 1, article e2555, 2017.

[13] Z. Jin, F. Liang, J. Yang, and W. Mei, "hnRNP I regulates neonatal immune adaptation and prevents colitis and colorectal cancer," PLoS Genetics, vol. 13, no. 3, article e1006672, 2017.

[14] Z. J. Zhou, Z. Dai, S. L. Zhou et al., "HNRNPAB induces epithelial-mesenchymal transition and promotes metastasis of hepatocellular carcinoma by transcriptionally activating SNAIL," Cancer Research, vol. 74, no. 10, pp. 2750-2762, 2014.

[15] Y. Yang, Q. Chen, H. Y. Piao et al., "HNRNPAB-regulated lncRNA-ELF209 inhibits the malignancy of hepatocellular carcinoma," International Journal of Cancer, vol. 146, no. 1, pp. 169-180, 2020.

[16] J. T. Hua, M. Ahmed, H. Guo et al., "Risk SNP-mediated promoter-enhancer switching drives prostate cancer through lncRNA PCAT19," Cell, vol. 174, no. 3, pp. 564-575.e18, 2018.

[17] Z. Tang, C. Li, B. Kang, G. Gao, C. Li, and Z. Zhang, "GEPIA: a web server for cancer and normal gene expression profiling and interactive analyses," Nucleic Acids Research, vol. 45, no. W1, pp. W98-W102, 2017.

[18] D. R. Rhodes, J. Yu, K. Shanker et al., "ONCOMINE: a cancer microarray database and integrated data-mining platform," Neoplasia, vol. 6, no. 1, pp. 1-6, 2004.

[19] P. Jézéquel, M. Campone, W. Gouraud et al., "bc-GenExMiner: an easy-to-use online platform for gene prognostic analyses in breast cancer," Breast Cancer Research and Treatment, vol. 131, no. 3, pp. 765-775, 2012.

[20] P. Jézéquel, J.-S. Frénel, L. Campion et al., "bc-GenExMiner 3.0: new mining module computes breast cancer gene expression correlation analyses," Database, vol. 2013, article bas060, 2013.

[21] J. Gao, B. A. Aksoy, U. Dogrusoz et al., "Integrative analysis of complex cancer genomics and clinical profiles using the cBioPortal," Science Signaling, vol. 6, no. 269, p. pl1, 2013.

[22] H. Mizuno, K. Kitada, K. Nakai, and A. Sarai, "PrognoScan: a new database for meta-analysis of the prognostic value of genes," BMC Medical Genomics, vol. 2, no. 1, 2009.

[23] A. Fabregat, S. Jupe, L. Matthews et al., "The Reactome pathway knowledgebase," Nucleic Acids Research, vol. 46, no. D1, pp. D649-D655, 2018.

[24] T. Geuens, D. Bouhy, and V. Timmerman, "The hnRNP family: insights into their role in health and disease," Human Genetics, vol. 135, no. 8, pp. 851-867, 2016. 